\title{
1-GHz pentacene diode rectifiers enabled by controlled film deposition on SAM-treated Au anodes
}

Chan-mo Kang, Jessica Wade, Sumin Yun, Jaehoon Lim, Hyunduck Cho, Jeongkyun Roh, Hyunkoo Lee, Sangwook Nam, Donal D. C. Bradley*, Ji-Seon Kim*, and Changhee Lee*

Prof. Changhee Lee, Dr. Chan-mo Kang, Mr. Sumin Yun, Dr. Jaehoon Lim, Dr. Hyunduck Cho, Mr. Jeongkyun Roh, Dr. Hyunkoo Lee, and Prof. Sangwook Nam

Department of Electrical and Computer Engineering and Inter-university Semiconductor

Research Center, Seoul National University, 1 Gwanak-ro, Gwanak-gu, Seoul, 08826, Korea

E-mail: chlee7@snu.ac.kr

Dr. Chan-mo Kang

IoT Convergence Research Department, Electronics and Telecommunications Research Institute, 218 Gajeong-ro, Yuseong-gu, Daejeon, 34129, Korea

Dr. Hyunkoo Lee

Soft I/O Interface Research Section, Electronics and Telecommunications Research Institute, 218 Gajeong-ro, Yuseong-gu, Daejeon, 34129, Korea

Prof. Ji-Seon Kim, Ms. Jessica Wade,

Department of Physics and Centre for Plastic Electronics,

Imperial College London, South Kensington Campus, London SW7 2AZ, UK

E-mail: ji-seon.kim@imperial.ac.uk;

Prof. Donal D. C. Bradley,

Departments of Engineering Science and Physics, Division of Mathematical, Physical and Life Sciences, University of Oxford, 9 Parks Road, Oxford OX1 3PD

E-mail: Donal.Bradley@imperial.ac.uk

Keywords: organic diodes; ultra-high frequency (UHF) organic rectifiers; pentacene molecular orientation; self-assembled monolayers; 2,3,4,5,6-pentafluorobenzenethiol (PFBT)

For automatic detection of objects for work efficiency and user convenience, low-cost radio frequency identification (RFID) tags, especially based on organic materials, have been extensively studied. ${ }^{[1-4]}$ One of the key elements in RFID tags is a rectifier, which supplies dc power to circuits by converting rf signal. Rectifiers based on organic diodes have been demonstrated at $13.56 \mathrm{MHz}$, which is the standard carrier frequency for so-called high frequency (HF) RFID systems. ${ }^{[5-7]}$ However, HF RFID systems have a short reading distance because they communicate by inductive coupling, which works in the near-field of the transmitter antenna. To achieve longer detection distances, RFID systems should be operated 
at ultra-high frequency (UHF, 860-960 MHz) in which range they can communicate by backscatter modulation. In addition, UHF RFID tags have other merits such as small antenna size and faster operation speed. Unfortunately, in spite of such advantages, realizing low-cost UHF RFID has been very difficult, mainly because of the difficulty in development of ultrafast rectifiers. Sani et al. reported all-printed diode operating at $1.6 \mathrm{GHz}$, but the diode was consisted of printed inorganic layers of $\mathrm{Si}$ and $\mathrm{NbSi}_{2}$ microparticles with an organic binder ${ }^{[8]}$ Because the operational frequency of the diode scales with its charge-transporting properties, the realization of the UHF rectifier based on organic materials has been a challenge. Recently, a rectifier with a 3-dB frequency reaching an impressive $700 \mathrm{MHz}$ in terms of voltage was demonstrated, but its voltage output $\left(V_{\text {out }}\right)$ at $1 \mathrm{GHz}$ was only $0.31 \mathrm{~V}$ for an AC input signal with $2 \mathrm{~V}$ amplitude. ${ }^{[9]}$

In order to achieve ultra-high frequency performance organic rectifiers, which commonly consist of diodes and capacitors, it is important to achieve high charge carrier injection efficiency and mobility within the organic semiconductor layer. Even if the work function of a metal electrode is selected to match the highest occupied molecular orbital (HOMO) level of an organic semiconductor, the formation of an adversely aligned dipole or other (e.g. oxide) interface layer can lead to a hole injection barrier, limiting charge injection. ${ }^{[10,11]}$ Selfassembled monolayers (SAMs) represent one good candidate for ensuring efficient charge injection by specifically tuning the metal work function. ${ }^{[12-15]}$ Interfacial charge trapping can also sometimes help. ${ }^{[16]}$ The permanent dipole moment of suitably selected SAM molecules changes the effective metal work function, reducing the charge injection barrier. SAMs may also be used to enhance the properties of gate dielectric layers in organic thin film transistors (TFTs). ${ }^{[17,18]}$ 
In addition to SAM-based metal work function tuning, surface energy characteristics are also altered by the SAM functional groups. This in turn can modify the subsequent deposition of organic semiconductor layers. In particular, pentacene grain formation, one of the important factors determining pentacene thin film mobility, is much affected by substrate surface energy. The SAM molecule functional groups can be selected to lower the surface energy, thereby enhancing molecular packing and improving mobility. ${ }^{[19]}$ Studies have shown that the orientation of pentacene deposited on $\mathrm{Au}$ is different to that deposited on SAM-treated $\mathrm{Au}{ }^{[20-}$ ${ }^{22]}$ The effect that such structural differences have on electrical characteristics for transport in the vertical direction (normal to the film plane) has not been investigated to any great extent; the great majority of studies have focused on in-plane transport within TFT structures. ${ }^{[23-25]}$ In this study, we investigated vertical diode structures instead of TFTs and as a result of the understanding gained we were able to fabricate ultrafast pentacene rectifiers with $V_{\text {out }}=3.8 \mathrm{~V}$ at $1 \mathrm{GHz}$ and with a $3 \mathrm{~dB}$ frequency, in terms of voltage, of $1.24 \mathrm{GHz}$, the highest value reported to date. ${ }^{[8]}$

Conjugated organic molecules such as pentacene, demonstrate strong electron-vibrational mode coupling with a dependence on orientation. This allows us to use Raman spectroscopy as a probe for molecular orientation. ${ }^{[26]}$ Here, a $514 \mathrm{~nm}$ excitation is employed to study the orientation of pentacene molecules on the gold substrate. The Raman-active vibrational modes of pentacene were identified using quantum chemical calculations. ${ }^{[26]}$ Strong modes corresponding to long- and short-axis vibrations of the pentacene core were measured at 1598 and $1372 \mathrm{~cm}^{-1}$, respectively. The intensity of Raman scattering from a particular mode is expected to be enhanced when the electric field of the excitation laser is parallel to the vibrational coordinate. Therefore the intensity ratio of the short- and long-axis vibrations can be used to identify the molecular orientation. On untreated gold substrates the intensity of the short-axis mode relative to the long-axis mode vibration was very low $(\approx 0.5)$, indicating 
molecules were lying horizontally in the plane of the substrate (Figure 1c). The ratio of shortto long-axis mode increased significantly after 2,3,4,5,6-pentafluorobenzenethiol (PFBT) treatment on gold, to $>7$, indicating the pentacene molecules were adopting a different packing structure, and standing more upright (see Figure 1d). The ratio of intensity of the short- to the long-axis modes does not vary for different thicknesses of pentacene films on untreated gold surfaces, whereas for PFBT treated surfaces it decreases from $\approx 9.0$ at $5 \mathrm{~nm}$ thickness to $\approx 6.5$ at a $75 \mathrm{~nm}$ thickness.

The pentacene film microstructure on bare and PFBT-coated Au was also analyzed by X-ray diffraction (XRD) in the reflection mode and atomic force microscopy (AFM) (see Figure S1 in Supporting Information (SI)). The XRD pattern for a 90-nm thickness pentacene film on PFBT-coated Au shows peaks at $5.73^{\circ}$ and $11.55^{\circ}$ with a corresponding interplanar $d$ spacing of $15.4 \AA$; these peaks can be assigned to the (001) and (002) planes, implying that the pentacene molecules stand up on PFBT-coated Au (see schematic in Figure 1). Conversely, the XRD pattern of a 90-nm thickness pentacene film on bare Au shows relatively weak peaks at larger $2 \theta$ values $19.25^{\circ}, 24.03^{\circ}$ and $28.21^{\circ}$ with corresponding interplanar $d$ spacings of 4.6 , 3.65, and $3.15 \AA$; these peaks can be assigned to the (110), (022) and (-201) planes, implying that pentacene molecules lie-down when deposited on bare Au. Such differences are also evident from AFM measurements. The pentacene films on PFBT-coated Au show more round grains whereas the pentacene films on bare Au show a more rod-like morphology, consistent with previous reports and with the proposed, respectively, standing up and lying down geometries for the pentacene molecules. ${ }^{[20-22]}$

The different pentacene microstructure on SAM-treated Au might be expected to influence the diode electrical properties. To investigate this, hole-only devices (HODs), comprising glass/Au/pentacene/ $\mathrm{MoO}_{3} / \mathrm{Al}$ and glass/Au/PFBT-SAM/pentacene/ $\mathrm{MoO}_{3} / \mathrm{Al}$, were fabricated, 
with the additional $\mathrm{MoO}_{3}$ layer included to ensure ohmic contact for hole injection from the $\mathrm{Al}$ contact. For bias voltages large enough to fill the traps (e.g. $>2 \mathrm{~V}$ for the HOD on PFBT-Au), the HOD current densities follow a quadratic dependence (see Figure S2 in SI), that can be modelled in terms of a space-charge limited current (SCLC) density:

$$
J_{S C L C}=\frac{9}{8} \mu \varepsilon_{0} \varepsilon_{r} \frac{V^{2}}{L^{3}}
$$

where $\mu$ is the mobility, $\varepsilon_{0}$ the vacuum permittivity, $\varepsilon_{r}$ the relative permittivity, $V$ the applied voltage, and $L$ the thickness of the active layer. A dielectric constant of 3.3, as obtained from the capacitance of pentacene diodes (see Figure S3 in SI) is used; this is similar to the previously reported value of 3.3-3.9 obtained by reverse-bias impedance-voltage measurements. ${ }^{[27]}$ From this equation, the through-plane diode mobilities for pentacene on $\mathrm{Au}$ and on PFBT-coated Au are calculated to be about $6.8 \times 10^{-4}$ and $1.1 \times 10^{-1} \mathrm{~cm}^{2} \mathrm{~V}^{-1} \mathrm{~s}^{-1}$, respectively. We note that the mobility extracted by a SCLC fit can be an underestimate due to non-ohmic injection and/or trapping effects. Therefore, to cross-check the deduced values, we also performed photoinduced charge carrier extraction by linearly increasing voltage (photo-CELIV) measurements (see Figure S4 in SI). From the photo-CELIV measurements, the through-plane hole mobility for pentacene films deposited on bare Au was deduced to be about $7.1 \times 10^{-4} \mathrm{~cm}^{2} \mathrm{~V}^{-1} \mathrm{~s}^{-1}$ at $2.95 \times 10^{4} \mathrm{~V} \mathrm{~cm}^{-1}$, in reasonable agreement with the $J_{S C L C}$ current density fit. However, for pentacene films deposited on PFBT-treated Au no photoCELIV peaks were obtained under the same experimental conditions. From the SCLC fit, the pentacene mobility on PFBT-treated Au is two orders of magnitude higher than that on bare $\mathrm{Au}$, which implies that the maximum current peak should occur in the sub- $\mu$ s range and, therefore, not be detectable with our measurement system. Unfortunately, time of flight photocurrent measurements require SCLC behaviour in substantially thicker films $(\approx$ few $\mu \mathrm{m})$ 
for which trapping effects are necessarily exacerbated ${ }^{[28-30]}$ and for which the microstructure may be different to that of the thin films of interest here for our diode structures. The alternative dark injection transient measurements ${ }^{[16]}$ require both ohmic injection and trap-free SCLC which may occur for pentacene films on PFBT-Au (where there is a small injection barrier due to the PFBT dipole moment (see below) and where the deduced SCLC mobility suggests low trap densities) but is not expected for pentacene films on bare Au. Such studies would make an interesting topic for future investigation but are beyond the scope of the current paper.

Our through-plane hole mobility measurements for pentacene films deposited on bare and PFBT-coated Au electrodes show that the standing-up molecular geometry of the latter is beneficial. The hole mobility of single crystalline pentacene along the $a b$ axis is known to be 5 times or more higher than along the $c$ axis, ${ }^{[31,32]}$ but thermally evaporated pentacene films are polycrystalline such that grain boundaries might be expected to play a dominant role in limiting transport. Assuming the same grain size, charge carriers in the pentacene film with lying down geometry are more likely to encounter grain boundaries in the vertical direction, leading to a decrease in through-plane mobility. Moreover, the pentacene film on bare $\mathrm{Au}$ exhibits rather weak XRD peaks, indicating that a relatively small fraction of material shows long range crystalline order. In addition, the pentacene on bare Au has a molecular tilt angle of about $30-45^{\circ}$ relative to the substrate plane, ${ }^{[22,33]}$ expected to result in randomly oriented pentacene grains. Figure 2 presents cross-sectional scanning electron microscope (SEM) images for pentacene on bare and PFBT-treated Au. Pentacene on bare Au does indeed exhibit a relatively disordered structure in comparison with pentacene on PFBT-treated Au for which a more dense and ordered structure is evident. It also appears that in the latter case grain boundaries are predominantly a feature of in-plane microstructure rather than throughplane where single grains span the full film thickness. 
Figure 3 shows (a) a schematic of the diode structure together with (b) an energy level diagram for the Au and Al electrodes, pentacene, and the PFBT SAM. Since Schottky diodes are more advantageous for high frequency operation than p-n junction diodes,${ }^{[34]}$ we sought to introduce a Schottky barrier at the pentacene/Al interface. We then also needed to create an Ohmic contact between the Au anode and pentacene semiconductor. This, despite the work functions of $\mathrm{Au}$ and pentacene being similar, is typically not formed due to disadvantageous interface dipoles that create an injection barrier of $\approx 0.5 \mathrm{eV} \cdot{ }^{[11,35]}$ Coating the Au electrode with the PFBT SAM significantly reduces the barrier and allows Ohmic contact.

Typical DC current density-voltage $(J-V)$ characteristics for pentacene diodes with both SAM-treated and bare Au anodes are presented in Figure 4. The data shows that the transition voltage, defined as the voltage at which the current changes from being ohmic to space charge limited, is substantially lower for diodes with PFBT-treated Au anodes $(\approx 0.1 \mathrm{~V})$ than for bare $\mathrm{Au}(\approx 1.1 \mathrm{~V})$, confirming the beneficial effect of SAM treatment on the injection barrier.

Previous reports show that the dipole moment of PFBT is $-1-2 \mathrm{D},{ }^{[36,37]}$ enabling a reduction in the injection barrier of $0.1 \mathrm{eV} \cdot{ }^{[38]}$ As a result, the forward-bias current density for the diode with PFBT-treated Au is much higher than that with bare $\mathrm{Au}$ and reaches $100 \mathrm{~A} \mathrm{~cm}^{-2}$ at $3 \mathrm{~V}$. In addition, the rectification ratio of the SAM-coated Au anode diodes, as shown in the inset to Figure 4 , is found to be $7.47 \times 10^{5}$ at $1 \mathrm{~V}$, and $1.05 \times 10^{7}$ at $2.8 \mathrm{~V}$, values that are between 1 and 3 orders of magnitude higher than previously reported for similar organic rectifying diodes. ${ }^{[7,8,39]}$ Rectification ratios of $2 \times 10^{5}$ at $1 \mathrm{~V}$ have previously been achieved for poly(3hexylthiophene):PCBM bulk heterojunction based diodes but required more complex device architectures. $^{[40]}$ The high rectification ratio achieved here is attributed to the combination of a very high forward bias current and moderately low reverse-bias leakage. Further 
enhancements might be anticipated through oxidation and SAM functionalization of the Al electrode leading to a further reduction in leakage current. ${ }^{[41]}$ In addition, large reverse-bias breakdown voltages, $V_{R}=18 \mathrm{~V}$, were measured for our diodes (see Figure $\mathrm{S} 5$ in $\mathrm{SI}$ ). In an ideal rectifier, the maximum reverse-bias voltage that can be applied to the diode should be more than twice the maximum input voltage. A high $V_{R}$ thus enables the rectifier to be used in high power circuits. Moreover, high input voltages can improve the frequency performance of the rectifier. ${ }^{[42]}$

Figure 5a shows the test setup used to characterize the frequency response of our pentacene diode rectifiers. It comprises a HP8657B signal generator fed through a power amplifier to the diode with a $51 \Omega$ input resistor, a $10 \mathrm{nF}$ load capacitor and a $1 \mathrm{M} \Omega$ load resistor. To minimise the rectifier output voltage ripple, the period of the input signal should be kept much smaller than the load RC time constant, $\tau_{\mathrm{RC}}$, and the load capacitance should be kept much larger than the capacitance of the diode. ${ }^{[42]}$ Satisfying the former condition is straightforward at high frequencies since $\tau_{\mathrm{RC}}=10 \mathrm{nF} \times 1 \mathrm{M} \Omega=10 \mathrm{~ms}$ but the latter condition, largely controlled by the diode area, needs to be carefully considered. In our case, the capacitance of the pentacene diode was measured to be only $1.7 \mathrm{pF}$, clearly satisfying the second condition. The diode area is additionally important in designing RFID tags that are more stable against breakdown; small area diodes can sustain larger voltages due to better heat dissipation, thus allowing higher rf power to be handled. ${ }^{[43]}$ The diode area chosen for this study was $80 \times 80 \mu \mathrm{m}^{2}$.

The frequency characteristics of a typical pentacene diode rectifier with a PFBT-treated Au anode are shown in Figure $\mathbf{5 b}$ for a sinusoidal peak-to-peak voltage input $V_{\text {in }}=10 \mathrm{~V}$. At the low $(1 \mathrm{MHz})$ end of the frequency range the output voltage $V_{\text {out }} \approx 6 \mathrm{~V}$, with the $\approx 4 \mathrm{~V}$ drop compared to $V_{\text {in }}$ due to power losses in the load resistor and from reverse-bias leakage currents. It is noted that the voltage drop in the rectifier is one of the important considerations. Long 
range electromagnetic coupling limits the input power and hence the voltage drop of the rectifier determines the capability of the UHF RFID tags. Ideally, the voltage drop is given by the difference between maximum amplitude of the input voltage and the turn-on voltage of the diode. For the actual device, however, the output voltage is additionally dropped by the power consumption of the load resistor, charge dissipation by leakage current of the diode and low forward bias current. From the simulation, we confirmed that almost $98.5 \%$ of discharge is attributed to the leakage current of the diode (see Figure S6 in SI). Thus, the voltage drop $\approx$ $4 \mathrm{~V}$ is dominantly caused by the leakage current of the diode. The aforementioned oxidation and SAM functionalisation of the Al electrode can lead to a further reduction in leakage current to minimize voltage drop. ${ }^{[41]}$ The large voltage drop also can be reduced by lowering the input voltage because it can effectively reduce the reverse bias applied to the diode. However, decreasing input voltage adversely affects 3-dB frequency. ${ }^{[42]}$ Unfortunately, the simulation results show that the optimal voltage range which satisfies both low voltage drop and high $3-\mathrm{dB}$ frequency does not exist because the $3-\mathrm{dB}$ frequency is monotonically increased as input voltage increases, whereas the voltage drop monotonically decreases as input voltage decreases (see Figure S7 in SI).

As the frequency increases, additional leakage current flows via the diode capacitance, decreasing $V_{\text {out }}$ further. If the diode is considered to comprise a single RC element, the expected frequency dependency of $V_{\text {out }}$ should be described by the following equation:

$$
V_{\text {out }}=\frac{V_{0}}{\sqrt{1+\left(f / f_{c}\right)^{2}}}
$$

where $V_{0}$ is the DC voltage output, $f$ the measurement frequency, and $f_{c}$ the cutoff frequency. By fitting the measured data as shown by the solid line in Figure $5 \mathrm{~b}, V_{0}$ and $f_{c}$ are deduced to 
be $6.02 \mathrm{~V}$ and $7.16 \times 10^{8} \mathrm{~Hz}$, respectively. Also, the $3-\mathrm{dB}$ frequency, in terms of voltage, which is often used to specify the frequency performance of rectifier diodes, ${ }^{[8,42]}$ is seen to be $1.24 \mathrm{GHz}$; the highest value reported for an organic rectifier diode to date ${ }^{[8]}$ Correspondingly, a relatively large $V_{\text {out }}=3.8 \mathrm{~V}$ is achieved at $1 \mathrm{GHz}$.

The excellent frequency performance of our rectifiers is attributed to the high performance pentacene diodes we have fabricated, with high mobiliy, injection efficiency, rectification ratio, and $V_{R}$ values. The through-plane mobility of pentacene films on PFBT-Au is significantly improved compared with films on bare Au. This is because, even though the pentacene film on PFBT-Au forms a polycrystalline structure, the associated grain boundaries are parallel to the charge transport direction (see Figure 2) leading to little consequent reduction in mobility. In addition, the PFBT dipole moment efficiently reduces the injection barrier, ensuring a high forward-bias current density that allows fast charging of the load capacitor for good frequency performance. Other desirable features of these pentacene diodes are their high rectification ratios and $V_{R}$ values. The former helps to block leakage current discharge of the diode thereby increasing the rectifier output voltage and the latter increases power handling capacity and allows a high $V_{\text {in }}$ that can further improve frequency response.

In summary, we have fabricated high performance UHF pentacene rectifiers by employing PFBT-coated Au as anode. From XRD, AFM and Raman analysis, the pentacene molecules on Au exhibit a lying-down orientation and those on PFBT-coated Au exhibit a standing-up orientation. The through-plane mobility of the pentacene film on PFBT-coated Au is, as a consequence, increased to $0.11 \mathrm{~cm}^{2} \mathrm{~V}^{-1} \mathrm{~s}^{-1}$. The PFBT SAM also lowers the injection barrier for hole injection, allowing the forward bias diode current density to reach $100 \mathrm{~A} \mathrm{~cm}^{-2}$ at $3 \mathrm{~V}$. The rectifier $3-\mathrm{dB}$ frequency, in terms of voltage, is $1.24 \mathrm{GHz}$ and $V_{\text {out }}=3.8 \mathrm{~V}$ at $1 \mathrm{GHz}$ is obtained for $V_{i n}=10 \mathrm{~V}$. This proof of concept study demonstrates the excellent potential that 
exists for low-cost, organic-semiconductor-based, flexible RFID tags. Our pentacene rectifiers show performance that is already sufficient for UHF use but before they can be deployed it will be necessary to address the susceptibility of pentacene thin films to oxygen and moisture degradation under ambient conditions. This is an especially significant problem for low-cost RFID applications where encapsulation is not an option. Nevertheless, because PFBT and similar dipolar SAMs are expected to be transferable for use with other p-type organic semiconductors, such as those currently being developed for high mobility transistors and for solar cells, we are optimistic that the future prospects for deployment of UHF organic RFID tags are good.

\section{Experimental Section}

Structural Characterization of Pentacene Films: Pentacene was purchased from the Tokyo Chemical Industry Co Ltd and used without further purification. Raman spectra were acquired using a Renishaw inVia commercial microscope fitted with a $514 \mathrm{~nm}$ Argon-ion excitation source $(50 \times$ objective $)$. For XRD and AFM measurements, $100-\mathrm{nm}$ thickness pentacene films were thermally deposited in vacuo at $3 \times 10^{-6}$ torr over a $1.2 \times 1.2 \mathrm{~cm}^{2}$ area onto silicon wafers with $300 \mathrm{~nm}$ of thermally grown oxide. XRD patterns of the pentacene films were obtained in the reflection mode using a PANalytical X'pert Pro diffractometer with $\mathrm{Cu} \mathrm{K \alpha}$ radiation $(\lambda=1.54 \AA)$. Background signals were removed by subtraction of XRD patterns obtained from $\mathrm{Si} / \mathrm{SiO}_{2}$ bare substrates. AFM images were obtained using a Park Systems XE100 microscope in non-contact mode using a silicon cantilever (ppp-NCHR, Nanosensors). The cross-section SEM images of 100-nm pentacene films prepared on SAM/gold with $\mathrm{n}++$ Si substrates were obtained using a JEOL JSM 6701F microscope.

Fabrication of Pentacene Diode Structures: Glass substrates were cleaned in an ultrasonic bath with acetone, isopropyl alcohol, and de-ionized water for 20 minutes in sequence. Then, 
50-nm-thickness of Au was thermally evaporated on top using a shadow mask to form four stripes of $80 \mu \mathrm{m}$ width and 1.8 to $4.4 \mathrm{~mm}$ length. Next, self-assembled monolayers were coated under ambient conditions onto the Au stripes by dipping the substrates for 2 minutes into $10 \mathrm{mM}$ toluene solutions of PFBT (purchased from Aldrich). After rinsing with toluene, the SAM-modified Au electrodes were dried in vacuo for 10 minutes and $90-120 \mathrm{~nm}$ of pentacene was evaporated on top. Finally, $100 \mathrm{~nm}$ thickness, $80 \mu \mathrm{m}$ width $\times 2 \mathrm{~mm}$ length $\mathrm{Al}$ stripes were evaporated orthogonal to the Au stripes as the top electrodes. The Au, pentacene, and aluminum layers were thermally deposited in vacuo at $3 \times 10^{-6}$ torr. The overlap between $\mathrm{Au}$ and $\mathrm{Al}$ electrodes defined the diode active area, here chosen to be $80 \times 80 \mu \mathrm{m}^{2}$. Before measurement, the diodes were encapsulated within an Ar-filled glove box using a UV resin (XNR 5570-B1, Nagase ChemteX Corp.). Additional diode structures, comprising glass/Au $(50 \mathrm{~nm}) / \mathrm{PFBT} /$ pentacene $(120 \mathrm{~nm}) / \mathrm{MoO}_{3}(10 \mathrm{~nm}) / \mathrm{Al}(100 \mathrm{~nm})$ and glass/Au $(50$ $\mathrm{nm}) /$ pentacene $(90 \mathrm{~nm}) / \mathrm{MoO}_{3}(10 \mathrm{~nm}) / \mathrm{Al}(100 \mathrm{~nm})$ were fabricated for space charge limited current density measurements of hole mobility. These devices are biased (Au at a negative potential relative to $\mathrm{Al}$ ) so as to inject holes from the aluminum contact. Pentacene diodes for photo-CELIV measurements comprising Au $(50 \mathrm{~nm}) /$ pentacene $(400 \mathrm{~nm}) / \mathrm{Al}(15 \mathrm{~nm})$ and $\mathrm{Au}$ $(50 \mathrm{~nm}) / \mathrm{PFBT} /$ pentacene $(400 \mathrm{~nm}) / \mathrm{Al}(15 \mathrm{~nm})$ were also fabricated.

Device Characterization: For photo-CELIV measurements, 580-nm wavelength Nd-Yag laser pulses (3-6 ns duration, $5 \mathrm{~Hz}$ repetition rate) were directed onto the thin Al cathodes of pentacene diode structures to generate free charge carriers. A function generator (AFG3101, Tektronix) was used to apply a linearly increasing voltage ramp with the time delay between the laser pulse and voltage controlled by using a photodiode signal to trigger the function generator. A digital oscilloscope (TDS054B, Tektronix) was used with a $50-\Omega$ load resistor to measure the photocurrent. $J-V$ diode characteristics were measured using a Keithley 236 source-measure unit under dark conditions. For frequency dependent rectification 
measurements samples were attached to a printed circuit board (PCB) with $50 \Omega$-designed microstrip lines. A $10-\mathrm{nF}$ load capacitor and $1 \mathrm{M} \Omega$ load resistor were also attached. A HP8657B signal generator with power amplifier (Empower RF Systems Inc. BBM1C4AEL) was then connected, using a $51-\Omega$ resistor on the PCB to define the rectifier $V_{\text {in }}$ and an oscilloscope (Tektronix TDS5104) to measure the rectifier output. Bayonet Neill-Concelman (BNC) and Sub-Miniature version A (SMA) cables were used to connect the components.

\section{Acknowledgements}

This work was supported by the Global Frontier R\&D Program on Center for Multiscale Energy System funded by the National Research Foundation under the Ministry of Science, ICT \& Future, Korea (grant number 2011-0031567). This work was also supported by the Human Resources

Development programme (No. 20124010203170) of the Korea Institute of Energy Technology Evaluation and Planning (KETEP), funded by the Ministry of Trade, Industry, and Energy, Korea. This work was also supported by the UK Engineering and Physical Sciences Research Council (EP/K029843/1 and DTA studentship) and Global Partnership Funding (GPF-14 175 Plastic Electronics) from the UK Science \& Innovation Network. DDCB acknowledges partial support from the UK Engineering and Physical Sciences Research Council via the "EPSRC Centre for Innovative Manufacturing in Large Area Electronics” (EP/K03099X/1).

\section{References:}

[1] E. Cantatore, T. C. T. Geuns, G. H. Gelinck, E. van Veenendaal, A. F. A. Gruijthuijsen, L. Schrijnemakers, S. Drews, and D. M. de Leeuw, IEEE J. Solid-State Circuit 2007, 42,84 .

[2] K. Myny, S. Van Winckel, S. Steudel, P. Vicca, S. De Jonge, M. J. Beenhakkers, C. W. Sele, N. A. J. van Aerle, G. H. Gelinck, J. Genoe, and P. Heremans, in IEEE International 
Solid-State Circuits Conference, ISSCC 2008, Digest of Technical Papers, San Francisco, USA, 3-7 February 2009, pp. 290-292.

[3] K. Myny, M. J. Beenhakkers, N. A. J. M. van Aerle, G. H. Gelinck, J. Genoe, W. Dehaene, and P. Heremans, in IEEE International Solid-State Circuits Conference, ISSCC 2009, Digest of Technical Papers, San Francisco, USA, 8-12 February 2009, pp. 206-207.

[4] K. Myny, S. Steudel, S. Smout, P. Vicca, F. Furthner, B. van der Putten, A. K. Tripathi, G. H. Gelinck, J. Genoe, W. Dehaene, and P. Heremans, Org. Electron. 2010, 11, 1176.

[5] S. Steudel, S. De Vusser, K. Myny, M. Lenes, J. Genoe, and P. Heremans, J. Appl. Phys. 2006, 99, 114519.

[6] Y. Ai, S. Gowrisanker, H. Jia, I. Trachtenberg, E. Vogel, R. M. Wallace, B. E. Gnade, R. Barnett, H. Stiegler, and H. Edwards, Appl. Phys. Lett. 2007, 90, 262105.

[7] C. Kang, Y. Hong, and C. Lee, Jpn. J. Appl. Phys. 2010, 49, 05 EB07.

[8] N. Sani, M. Robertsson, P. Cooper, X. Wang, M. Svensson, P. A. Ersman, P. Norberg, M. Nilsson, D. Nilsson, X. Liu, H. Hesselbom, L. Akesso, M. Fahlman, X. Crispin, I. Engquist, M. Berggren, and G. Gustafsson, Proc. Natl. Acad. Sci. USA 2014, 111, 11943.

[9] D. Im, H. Moon, M. Shin, J. Kim, and S. Yoo, Adv. Mater. 2011, 23, 644.

[10] N. Koch, A. Kahn, J. Ghijsen, J. J. Pireaux, J. Schwartz, R. Johnson, and A. Elschner, Appl. Phys. Lett. 2003, 82, 70.

[11] L. Diao, C. D. Frisbie, D. D. Schroepfer, and P. P. Ruden, J. Appl. Phys. 2007, 101, 014510 .

[12] I. Campbell, J. Kress, R. Martin, D. Smith, N. Barashkov, and J. Ferraris, Appl. Phys. Lett. 1997, 71, 3528.

[13] D. J. Gundlach, J. Li Li, and T. N. Jackson, IEEE Electron Device Lett. 2001, 22, 571.

[14] S. Khodabakhsh, D. Poplavskyy, S. Heutz, J. Nelson, D. D. C. Bradley, H. Murata, and T. S. Jones, Adv. Funct. Mater. 2004, 14, 1205.

[15] J. P. Hong, A. Y. Park, S. Lee, J. Kang, N. Shin, and D. Y. Yoon, Appl. Phys. Lett. 2008, 92, 143311. 
[16] D. Poplavskyy, J. Nelson, and D. D. C. Bradley Appl.Phys.Lett. 2003, 83, 707.

[17] P. H. Wöbkenberg, J. Ball, F. B. Kooistra, J. C. Hummelen, D. M. de Leeuw, D. D. C. Bradley, and T. D. Anthopoulos, Appl.Phys.Lett. 2008, 93, 013303

[18] J. G. Labram, P. H. Wöbkenberg, D. D. C. Bradley, and T. D. Anthopoulos, Org. Electron. 2010, 11, 1250.

[19] S. S. Yang, K. Shin, and C. E. Park, Adv. Funct. Mater. 2005, 15, 1806.

[20] W. S. Hu, Y. T. Tao, Y. J. Hsu, D. H. Wei, and Y. S. Wu, Langmuir 2005, 21, 2260.

[21] N. Koch, A. Elschner, J. Schwartz, and A. Kahn, Appl. Phys. Lett. 2003, 82, 2281.

[22] D. Käfer, L. Ruppel, and G. Witte, Phys. Rev. B 2007, 75, 085309.

[23] D. T. James, B. K. C. Kjellander, W. Smaal, G. H. Gelinck, C. Combe, I. McCulloch, R. Wilson, J. H. Burroughes, D. D. C. Bradley, and J.-S. Kim, ACS Nano 2011, 5, 9824.

[24] J. Smith, R. Hamilton, M. Heeney, D. M. de Leeuw, E. Cantatore, J. E. Anthony, I. McCulloch, D. D. C. Bradley, and T. D. Anthopoulos, Appl. Phys. Lett. 2008, 93, 253301.

[25] R. Hamilton, J. Smith, S. Ogier, M. Heeney, J. E. Anthony, I. McCulloch, J. Veres, D.

D. C. Bradley, T. D. Anthopoulos, Adv. Mater. 2009, 21, 1166.

[26] D. T. James, J. M. Frost, J. Wade, J. Nelson, and J.-S. Kim, ACS Nano 2013, 7, 7983.

[27] C. H. Kim, O. Yaghmazadeh, D. Tondelier, Y. B. Jeong, Y. Bonnassieux, and G. Horowitz, J. Appl. Phys. 2011, 109, 083710.

[28] B. K. Yap, R. Xia, M. Campoy-Quiles, P. N. Stavrinou, D. D. C. Bradley, Nature Materials 2008, 7, 376.

[29] T. Kreouzis, D. Poplavskyy, S. M. Tuladhar, M. Campoy-Quiles, J. Nelson, A. J. Campbell, D. D. C. Bradley Phys. Rev. 2006, B73, 235201.

[30] A. J. Campbell, D. D. C. Bradley, H. Antoniadis, M. Inbasekaran, W. W. Wu and E. P. Woo, Appl.Phys.Lett. 2000, 76, 1734.

[31] Gilles A. de Wijs, Christine C. Mattheus, Robert A. de Groot, and Thomas T.M. Palstra, Synth. Met. 2003, 139, 109. 
[32] N. Ohashi, H. Tomii, M. Sakai, K. Kudo, and M. Nakamura, Appl. Phys. Lett. 2010, 96, 203302.

[33] Y. Zheng, D. Qi, N. Chandrasekhar, X. Gao, C. Troadec, and A. T. S. Wee, Langmuir 2007, 23, 8336 .

[34] R. F. Pierret, in Semiconductor device fundamentals, Addison-Wesley, New York, 1996.

[35] F. Amy, C. Chan, and A. Kahn, Org. Electron. 2005, 6, 85.

[36] C. Schmidt, A. Witt, and G. Witte, J. Phys. Chem. A 2011, 115, 7234.

[37] K. A. Singh, T. L. Nelson, J. A. Belot, T. M. Young, N. R. Dhumal, T. Kowalewski, R. D. McCullough, P. Nachimuthu, S. Thevuthasan, and L. M. Porter, ACS Appl. Mater. Interfaces 2011, 3, 2973.

[38] Z. Jia, V. W. Lee, I. Kymissis, L. Floreano, A. Verdini, A. Cossaro, and A. Morgante, Phys. Rev. B 2010, 82, 125457.

[39] K. Myny, S. Steudel, P. Vicca, J. Genoe, and P. Heremans, Appl. Phys. Lett. 2008, 93, 093305.

[40] L. Chen, P. Degenaar, D. D. C. Bradley, Adv.Mater. 2008, 20, 1679.

[41] J. M. Ball, P. H. Wöbkenberg, F. Colléaux, M. Heeney, J. E. Anthony, I. McCulloch, D. D. C. Bradley, T. D. Anthopoulos, Appl. Phys. Lett. 2009, 95, 103310.

[42] S. Steudel, K. Myny, V. Arkhipov, C. Deibel, S. De Vusser, J. Genoe, and P. Heremans, Nat. Mater. 2005, 4, 597.

[43] S. Kim, H. Cho, Y. Hong, and C. Lee, Mol. Cryst. Liquid Cryst. 2009, 513, 256. 
(a)

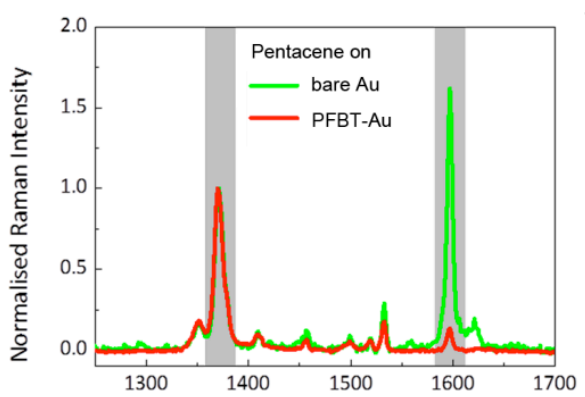

(b)

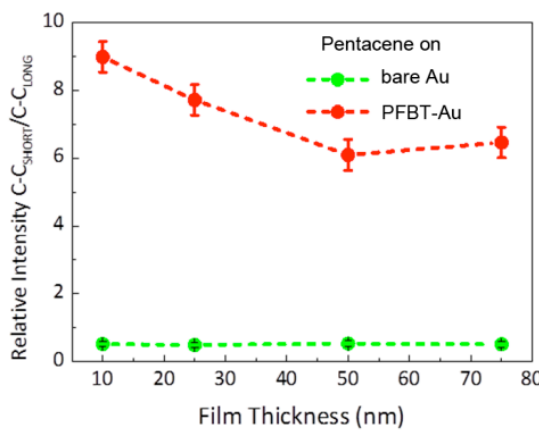

(c)

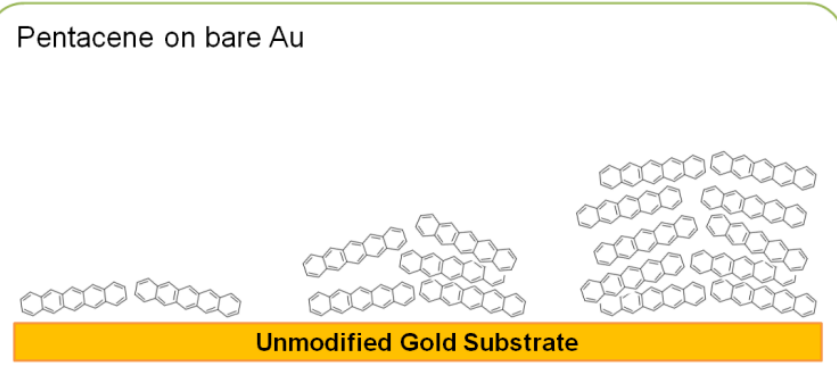

Penacene on PFBT-Au

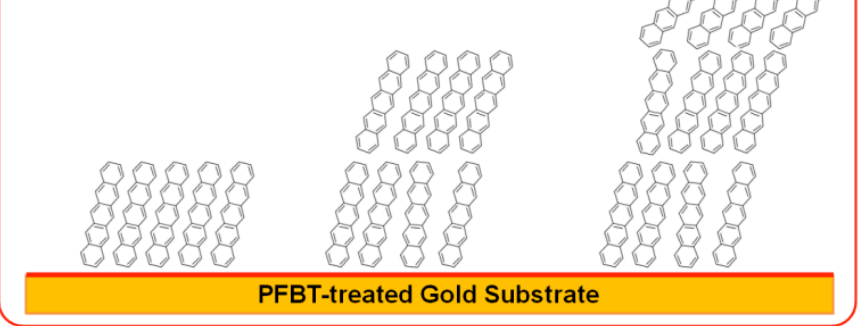

Figure 1. (a) Raman spectra of pentacene thin films on gold, with and without the PFBT SAM; intensities are normalised to the $1372 \mathrm{~cm}^{-1}$ short axis vibrational mode, (b) Intensity ratio of $1372 \mathrm{~cm}^{-1}$ and $1598 \mathrm{~cm}^{-1}$ vibrational modes as a function of pentacene film thickness and schematic molecular packing structures of pentacene molecules on (c) unmodified and (d) SAM-treated gold-coated substrates. 

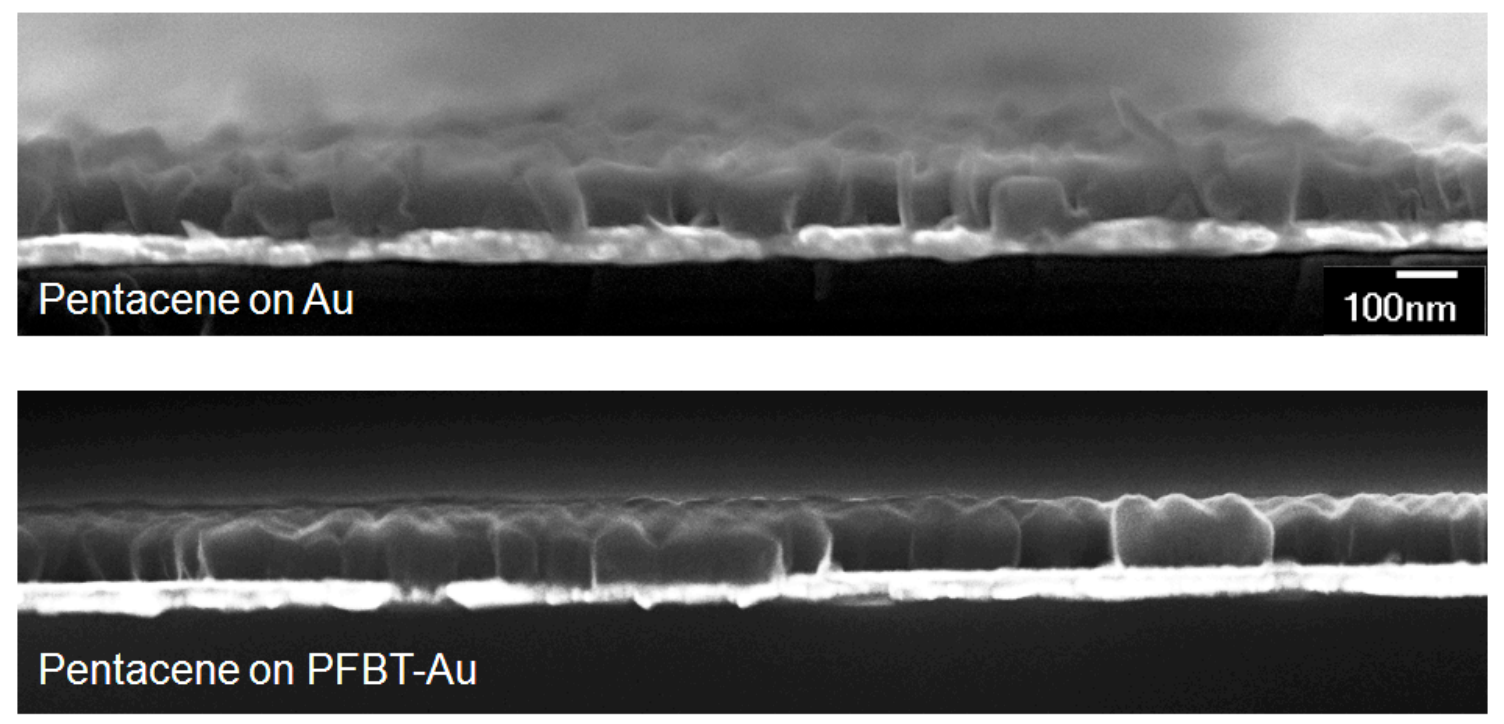

Figure 2. Cross-section SEM images of 100-nm thick pentacene films thermally evaporated at 3-4 $\AA^{-1}$ onto bare Au (upper image) and PFBT-coated Au (lower image). The scale bar indicates $100 \mathrm{~nm}$. 
(a)

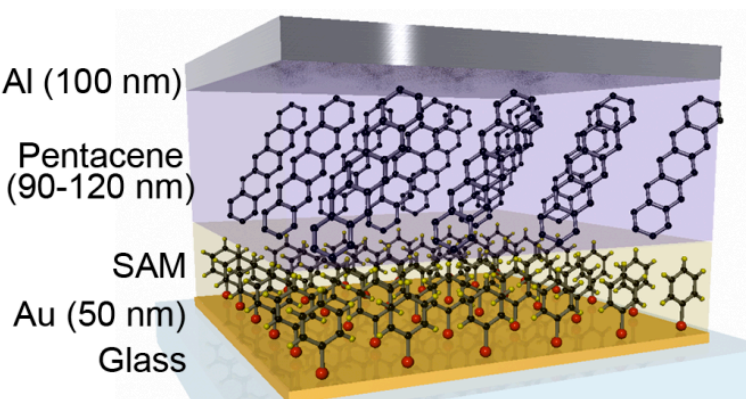

(b)

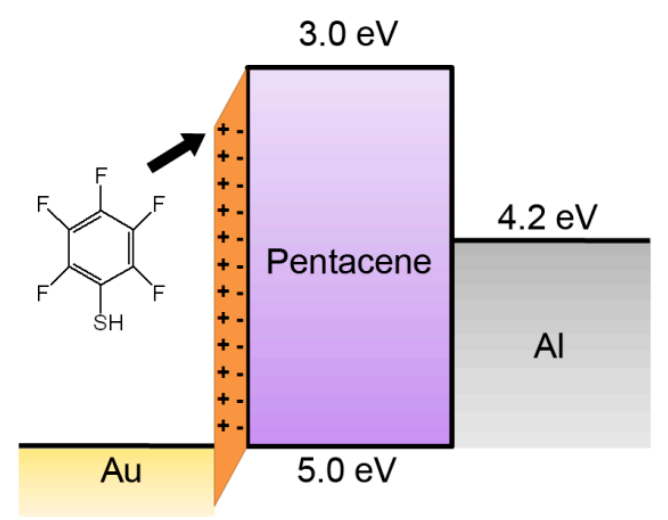

Figure 3. (a) Schematic structure of a pentacene diode with a PFBT-coated Au anode. For SCLC current density measurements of hole mobility, an additional $\mathrm{MoO}_{3}$ electron-blocking layer was inserted beneath the Al top electrode and for photo-CELIV in addition to the $\mathrm{MoO}_{3}$ layer, the pentacene film thickness was increased to $400 \mathrm{~nm}$. Reference diode structures were also fabricated without the PFBT SAM. (b) Corresponding energy level diagram of pentacene diodes with PFBT-coated Au. The PFBT chemical structure is also shown. 


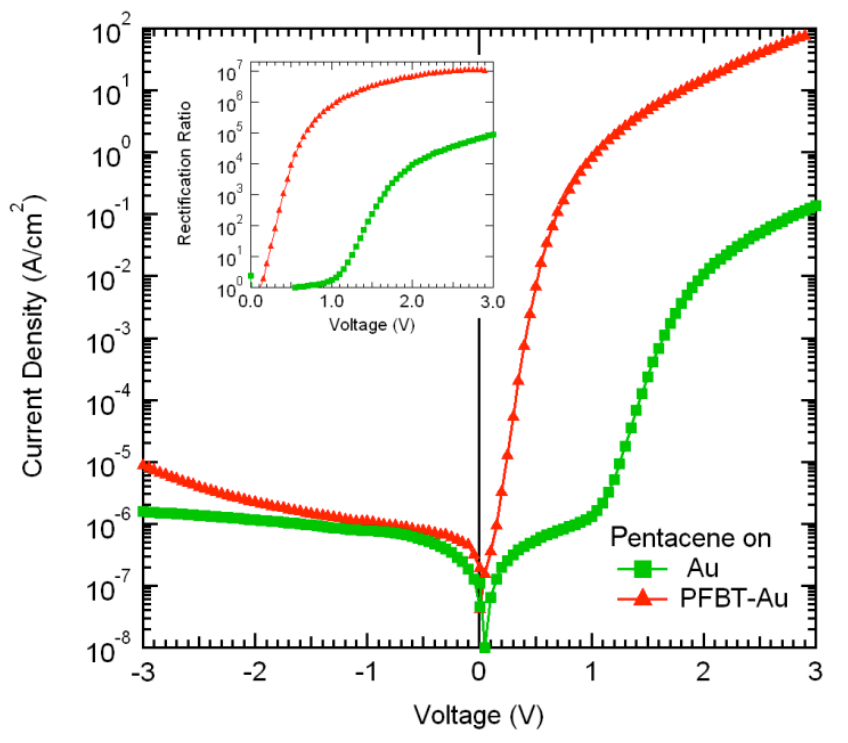

Figure 4. DC current density-voltage characteristics for pentacene diodes both with (filled triangles) and without (filled squares) a PFBT SAM coating on the Au anode. The pentacene films were thermally evaporated with a relatively high deposition rate $\left(3-4 \AA s^{-1}\right)$ in order to prevent shunts. ${ }^{[38]}$ These diodes had an active area, defined by the overlap of Au and Al electrodes, of $6400 \mu \mathrm{m}^{2}$. The inset shows the corresponding diode rectification ratios. 
(a)

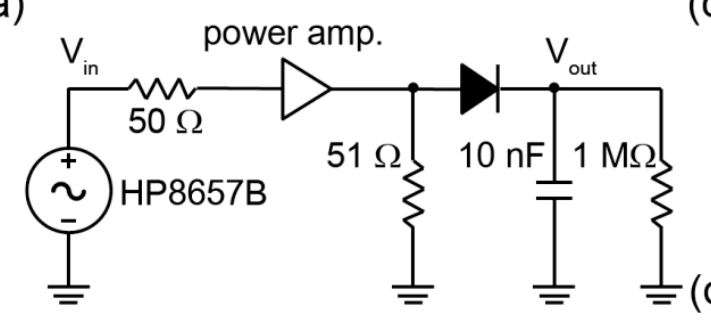

(b)

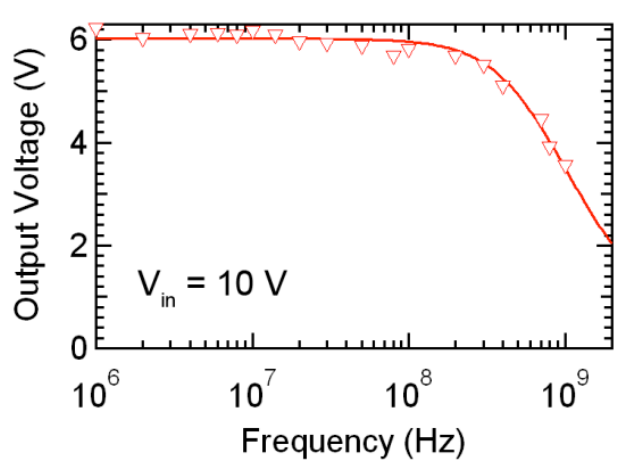

(c)

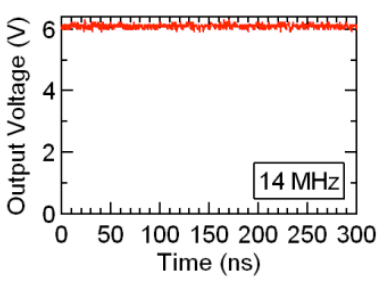
Time (ns)

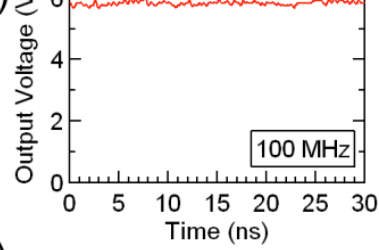

(e)

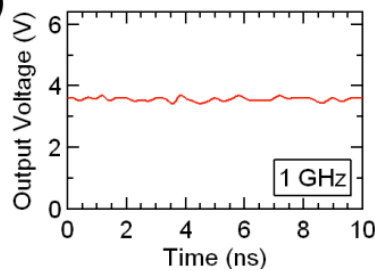

Figure 5. (a) Equivalent circuit for the rectifier frequency dependence measurement system. (b) Frequency characteristics for a PFBT-coated Au anode pentacene diode rectifier with a 10 $\mathrm{V}$ peak-to-peak sinusoidal input voltage. The solid line represents a fit to equation (2) - see text for further details. The time-dependent output voltages of PFBT-coated Au anode pentacene rectifiers operating at (c) $14 \mathrm{MHz}$, (d) $100 \mathrm{MHz}$, and (e) $1 \mathrm{GHz}$ are also shown. 
Table 1. Electrical parameters for pentacene diodes with bare and SAM-coated Au anodes.

\begin{tabular}{ccccc}
\hline & $\begin{array}{c}\text { Transition } \\
\text { voltage (V) }\end{array}$ & $\begin{array}{c}\text { Rectification } \\
\text { ratio at 3 V }\end{array}$ & $\begin{array}{c}\text { Hole Mobility } \\
\text { (SCLC) } \\
{\left[\mathbf{c m}^{2} \mathbf{~ V}^{-1} \mathbf{s}^{-1}\right]}\end{array}$ & $\begin{array}{c}\text { Hole Mobility } \\
\text { (photo-CELIV) }\end{array}$ \\
\hline Bare Au & 1.1 & $8.81 \times 10^{4}$ & $6.8 \times 10^{-4}$ & $7.1 \times 10^{-4}$ \\
Au/PFBT & 0.1 & $1.05 \times 10^{7}$ & $\left.1.1 \times 10^{-1} \mathbf{s}^{-1}\right]$ & - \\
\hline
\end{tabular}

The hole mobility data were determined for diodes that had an additional $\mathrm{MoO}_{3}$ electron blocking layer beneath the Al cathode. No photo-CELIV transients were resolved for the $\mathrm{Au} / \mathrm{PFBT}$ structures. The transition voltage is defined as the voltage at which the current changes from Ohmic to space charge limited transport. See text for further details. 